\title{
Im Gesundheitswesen geht sehr viel schief
}

\author{
Das Klima im Behandlungsteam beeinflusst die Patientensicherheit
}

Etwa 60 Prozent der Schadensfälle in einem Krankenhaus werden im OP verursacht. Ein gut eingespieltes Team und der Einsatz einer Checkliste reduzieren laut einer WHO-Studie aus dem Jahr 2009 Komplikationen in diesem Bereich um etwa 30 Prozent. Allerdings: Diese Ergebnisse wurden in der Folge nicht besonders breit diskutiert und eine flächendeckende Umsetzung in den Alltag ist ausgeblieben, konstatiert Univ.-Prof. Dr. Norbert Pateisky, Leiter der Abteilung für klinisches Risikomanagement und Patientensicherheit an der Universitätsfrauenklinik Wien. Die Rolle des Teams in Sachen Patientensicherheit stand kürzlich im Mittelpunkt des 20. АВBOTT Medical Circle in Wien.

„Wie wir wissen, geht im Gesundheitswesen sehr viel schief“, erklärte Patientenanwaltsprecher Dr. Gerald Bachinger. Neben Fehlern im OP geht es dabei auch um die Medikamentensicherheit und die Verordnungsqualität, sowohl im Spital als auch im niedergelassenen Bereich. „Einer Studie aus Salzburg aus 2009 zufolge“, so Bachinger, „kommen 50 Prozent der Patienten über 60 Jahren wegen schlechter Verordnungsqualität ins Krankenhaus.“ Die Verschreibung von zu vielen Medika- menten oder Medikamenten, die ihre Wirkung gegenseitig negativ beeinflussen, könnten mit dem Instrument der E-Medikation deutlich reduziert werden. Derzeit gibt es jedoch vor allem von Seiten der Ärzte Vorbehalte. Die Konsequenzen aus Fehlmedikationen sind allerdings glücklicherweise meist weniger dramatisch: Nur in fünf Prozent dieser Fehler komme es, so Pateisky, zu einem Schaden. Auch die Schnittstelle zwischen niedergelassenem Arzt und Krankenhaus bedürfe jedenfalls einer Verbesserung, stellte Bachinger fest.

Alle Fehler kann man nie vermeiden, meinte Pateisky, das Ziel von Risikomanagement ist aber, keinen Schaden zu erzeugen, indem Fehler im Ansatz erkannt, beendet und möglichst behoben werden. Dies sei nachgewiesenermaßen in einem guten Teamklima wesentlich besser möglich, als bei Spannungen im Team. Daher ist das gemeinsame Training des Teams von großer Bedeutung, auch um den gemeinsamen Umgang mit der Checkliste zu üben. „Anordnen von oben nützt nichts“, so Pateisky: „Das wichtigste ist, dass das Team zum Team wird. Dazu benötigt es Training und Schulung." Es gehe dabei, so Bachinger, ebenso um die emotionale Ebene und eine offene Fehlerkultur - auch gegenüber dem Patienten. Es sei nicht ein- zusehen, dass dieses wichtige Instrument der Qualitätssicherung aus finanziellen Gründen nicht eingesetzt und damit der Patient gefährdet werde. „Das ist auch eine ethische und rechtliche Frage“, unterstrich Pateisky.

Als alternatives Instrument, mögliche Fehlerquellen zu reduzieren, nannte Univ.Prof. Dr. Stefan Kriwanek, Vorstand Chirurgische Abteilung, SMZ Ost Donauspital, Wien, das Risikoassessment. In jedem Fall gelte es ein Risikobewusstsein zu schaffen. Die Motivation, sich mit dem Thema Risikomanagement auseinanderzusetzen, sei von Abteilung zu Abteilung unterschiedlich. Qualitätskriterien werden in der Dokumentation in österreichischen Spitälern derzeit - abgesehen von Sterblichkeit allgemein und der Reinterventionsrate in der Chirurgie - nicht erfasst. Eine Vergleichbarkeit ist daher schwer möglich.

„Wahrscheinlich gibt es kein allgemein gültiges bestes System“, resümmierte Pateisky: „Unsere Aufgabe ist aber, den besten Österreichmix zusammenzustellen."

Quelle: 20. ABBOTT Medical Circle Jubiläumsveranstaltung - Gesprächsrunde, „,Medical Team Training" - der Golden Standard in Sachen Patientensicherheit?, 20. Juni 2011, Wien

\section{S3-Prostatakarzinom-Leitlinie}

\section{Aktualisierung online}

Fast genau zwei Jahre nach ihrer Veröffentlichung wird die „Interdisziplinäre Leitlinie der Qualität S3 zur Früherkennung, Diagnose und Therapie der verschiedenen Stadien des Prostatakarzinoms" aktualisiert. Mit der Aktualisierung verfolgen die beteiligten Deutschen Fachgesellschaften ihr Konzept der „lebendigen Leitlinie“, das neue wissenschaftliche Erkenntnisse möglichst schnell in der medizinischen Versorgung verankern soll.

Bei der Aktualisierung wurde jenen Themen Priorität eingeräumt, für die eine mögliche Veränderung von Empfehlungen gesehen wurde, wie etwa bei der Früherkennung, der Behandlung des kastrationsresistenten Prostatakarzinoms oder der Behandlung von Knochenmetastasen. Aber auch neue Themen wurden bearbeitet. Die Leitlinie enthält nun detaillierte Empfehlungen, welche bildgebende Diagnostik für welche Indikation in Primär- und Ausbreitungsdiagnostik empfohlen wird - aber auch, was nicht empfohlen wird. Für die Behandlung von Knochenmetastasen und insbesondere für die Zweitlinientherapie des kastrationsresistenten Prostatakarzinoms werden neue medikamentöse Optionen genannt, aber auch die Prävention von Komplikationen angesprochen.
Für die aktive Überwachung (Active Surveillance) wurden die Indikationen eines Abbruchs der Therapie präzisiert. Bei der Strahlentherapie des high risk bzw. lokal fortgeschrittenen Karzinoms wird auch die Option einer kürzeren Hormontherapie als bisher eingeräumt. Bei der Früherkennung hat sich dagegen lediglich das Statement zur Studienlage, nicht aber das grundsätzliche Vorgehen geändert.

Informationen: www.urologenportal.de

Quelle: Presseinformation des Berufsverbands der Deutschen Urologen e.V. und der Deutschen Gesellschaft für Urologie e.V 